\title{
P06-06. Capacity building for HIV vaccine trials in Africa through
} South-South collaboration

\author{
J Mpendo*1, V Mwapasa², A Kamali³, J Seeley³, J Birungi ${ }^{1}$, H Njai ${ }^{3}$, \\ A Ssemaganda ${ }^{1}$, J de Bont ${ }^{1}$, T Mebrahtu ${ }^{1}$, A Nanvubya ${ }^{1}$, G Asiki ${ }^{3}$, E Kintu ${ }^{4}$, \\ M Moore $^{2}$ and P Kaleebu ${ }^{4}$
}

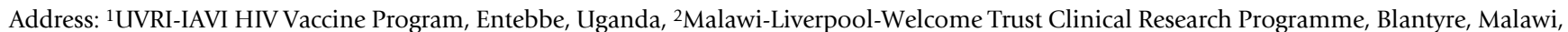 \\ ${ }^{3}$ Medical Research Council/UVRI Uganda Research Unit on AIDS, Entebbe, Uganda and ${ }^{4}$ Uganda Virus Research Institute, Entebbe, Uganda \\ * Corresponding author
}

from AIDS Vaccine 2009

Paris, France. 19-22 October 2009

Published: 22 October 2009

Retrovirology 2009, 6(Suppl 3):P95 doi:10.1186/1742-4690-6-S3-P95

This abstract is available from: http://www.retrovirology.com/content/6/S3/P95

(C) 2009 Mpendo et al; licensee BioMed Central Ltd.

\section{Background}

Sub-Saharan Africa (SSA) remains the region most affected by HIV. A safe, effective and accessible vaccine is required to complement the existing HIV/AIDS prevention strategies. Until recently, HIV vaccine research was conducted mainly in the developed world, but increasingly is taking place in developing countries. In recent years capacity for such research has been developed in SSA through the support of international partners. With the growing mandate to conduct HIV prevention research in Africa, there is need to sustain and complement this capacity building by supporting South-South networks to strengthen the existing experience and expertise.

\section{Methods}

Through a 3 year grant from the European and Developing Countries Clinical Trials Partnerships (EDCTP) and with the support of partners such as the International AIDS Vaccine Initiative (IAVI), South-South collaboration has been established between the Uganda Virus Research Institute (UVRI) and the Malawi-Liverpool-Welcome Trust Clinical Research Programme (MLW). The main focus of this collaboration is on cohort development for future HIV vaccine trials, technology transfer, training in Good Clinical Practice (GCP) and Good Clinical Laboratory Practice (GCLP) and social science studies and project management methodologies. Common research goals have been identified and implemented via exchange vis- its, conference calls and participation in training workshops, creating a strong South-South synergy.

\section{Results}

Partners collaborated to develop similar protocols to recruit high risk individuals from fishing communities of Lake Victoria and Lake Malawi into a prospective study integrating epidemiology, social science and virology. Methodologies for the recruitment and retention of these marginalized populations are shared between partners and will hopefully lead to great potential for future HIV vaccine research.

\section{Conclusion}

We have observed that South-South experience and capacity sharing is mutually beneficial and supports African scientists in the conduct of relevant research in SSA for the benefit of the global HIV vaccine effort. 